\title{
SELENATO E SELENITO NA PRODUÇÃO, NUTRIÇÃO MINERAL E BIOFORTIFICAÇÃO COM SELÊNIO EM CULTIVARES DE ALFACE ${ }^{(1)}$
}

\author{
Sílvio Júnio Ramos ${ }^{(2)}$, Valdemar Faquin ${ }^{(3)}$, Hilário Júnior de Almeida(4), \\ Fabrício William Ávila ${ }^{(5)}$, Luiz Roberto Guimarães Guilherme ${ }^{(6)}$, Carla \\ Elisa Alves Bastos ${ }^{(7)} \&$ Patrícia Andressa Ávila ${ }^{(8)}$
}

\section{RESUMO}

O selênio (Se) é um importante elemento ligado a processos físiológicos na planta, microrganismos, animais e seres humanos. No entanto, para as plantas, seu modo de ação e sua essencialidade são ainda motivos de controvérsia. No Brasil, é relevante a falta de informações sobre o Se nas culturas agrícolas, havendo ainda indicativo de baixa ingestão desse elemento pela população. Assim, este estudo objetivou avaliar a influência da aplicação de selenato e selenito na biofortificação com Se e o efeito dessas formas de Se nos teores de macro e micronutrientes em cultivares de alface. Utilizou-se o delineamento experimental inteiramente casualizado, em esquema fatorial $5 \times 3 \times 2$, sendo cinco cultivares de alface (Maravilha de Verão, Rafaela, Great Lakes, Veneranda e Vera), três concentrações de Se (0, 10 e $\left.20 \mu \mathrm{mol} \mathrm{L} \mathrm{L}^{-1}\right)$ e duas formas de Se (selenato e selenito), com quatro repetições. Os resultados encontrados mostram que, para o cultivo de alface em solução nutritiva, o selenato foi mais indicado para a biofortificação com Se, enquanto o selenito mostrou ser mais tóxico. A aplicação do selenato resultou em aumento no teor de $\mathrm{S}$ na parte aérea, enquanto o selenito reduziu o teor de $\mathrm{P}$, e ambas as formas de Se diminuíram os teores de micronutrientes. Entre as cultivares de alface, não se observou variação genotípica para o teor de Se, e pequena variação foi verificada para produção de matéria seca da parte aérea e teores de $S$, Mg, Mn e Fe.

Termos de indexação: Lactuca sativa, teor de selênio, micronutriente, macronutriente.

\footnotetext{
(1) Parte da Dissertação de Mestrado do terceiro autor. Recebido para publicação em 14 de julho de 2010 e aprovado em 14 de abril de 2011.

(2) Pós-Doutorando em Ciência do Solo, Departamento de Ciência do Solo, Universidade Federal de Lavras - UFLA. Caixa Postal 3037, CEP 37200-000 Lavras (MG). Bolsista DTI-A do CNPq. E mail: silviojramos@dcs.ufla.br

(3) Professor Titular do Departamento de Ciência do Solo, UFLA. Bolsista do CNPq. E-mail: vafaquin@dcs.ufla.br

(4) Doutorando em Ciência do Solo, Faculdade de Ciências Agrárias e Veterinárias, Universidade Estadual Paulista "Julio de Mesquita Filho". CEP 14884-900 Jaboticabal (SP). E mail: hilarioagro@yahoo.com.br

(5) Doutorando em Ciência do Solo, Departamento de Ciência do Solo, UFLA. Bolsista do CNPq. E mail: avilafw@yahoo.com.br

(6) Professor Associado do Departamento de Ciência do Solo, UFLA. Bolsista do CNPq. E mail: guilherm@dcs.ufla.br

(7) Mestranda em Solos e Nutrição de Plantas, Escola Superior de Agricultura “Luiz de Queiroz", Universidade de São Paulo. CEP 13418-900 Piracicaba (SP). E mail: ceab@ymail.com

(8) Graduanda em Engenharia Florestal, UFLA. Bolsista de I.C./CNPq E mail: andressapa@yahoo.com.br
} 


\title{
SUMMARY: SELENATE AND SELENITE ON YIELD, MINERAL NUTRITION AND BIOFORTIFICATION WITH SELENIUM IN LETTUCE CULTIVARS
}

\begin{abstract}
Selenium is an important antioxidant element associated with physiological processes in plants, microorganisms, animals, and humans. However, its mode of action and essentiality in plants are still disputed. In Brazil, information on Se in agricultural crops is extremely scarce, though there are indications that low levels of Se are being consumed by the population. The objective of this study was to evaluate the effect of selenate and selenite on yield, mineral nutrition and biofortification with Se of lettuce cultivars. The experiment was arranged in a completely randomized factorial design $5 \times 3 \times 2$, with five lettuce cultivars (Maravilha de Verão, Rafaela, Great Lakes, Veneranda, and Vera), three Se concentrations (0, 10 and $20 \mu \mathrm{mol} \mathrm{L} \mathrm{L}^{-1}$ ) and two forms of Se (selenate and selenite), with four replicates. Results indicate that selenate application is more appropriate for biofortification of lettuce cultivars, while the effect of selenite proved to be more toxic. The application of selenate results in increased $S$ shoot concentrations, while selenite reduced $P$ concentrations, and both Se forms decreased micronutrient concentrations. No genotypic variation among lettuce cultivars was observed for Se concentration, and little variation was observed for shoot dry matter yield and $\mathrm{S}, \mathrm{Mg}$, $\mathrm{Mn}$, and Fe levels.
\end{abstract}

Index terms: Lactuca sativa, selenium concentration, micronutrient, macronutrient.

\section{INTRODUÇÃO}

O selênio (Se) é essencial para o metabolismo humano e exerce atividade biológica por meio de sua incorporação em proteínas, formando as selenoproteínas, que desempenham atividade antioxidante capaz de reduzir peróxidos no corpo humano (Rayman, 2002). Além disso, atividade anticancerígena de algumas formas de Se no cólon, pulmão, pele e em outros tipos de câncer tem sido demonstrada (Stratton et al., 2003; Fairweather-Tait et al., 2011). Entretanto, a essencialidade desse elemento para as plantas ainda é motivo de controvérsia no meio científico (Zhu et al., 2009; PilonSmits \& Quinn, 2010). Há relatos que o Se pode ajudar as plantas a se manterem por mais tempo fisiologicamente ativas, aumentando a produção vegetal (Hartikainen et al., 2000; Lyons et al. 2009; Ramos et al., 2011).

A interação entre o Se e outros elementos químicos é principalmente documentada para humanos e animais. Para as plantas, pouca atenção é dada ao efeito desse elemento na absorção e nos teores de macro e micronutrientes. Alguns estudos têm mostrado que o Se compete com o P no processo de absorção (Hopper \& Parker, 1999) e aumenta a absorção de S (White et al., 2004), além de utilizar os mesmos transportadores e rota de assimilação deste último (Pilon-Smits \& Quinn, 2010).

Com relação à biofortificação com Se, essa técnica consiste basicamente em aumentar a concentração desse elemento em culturas agrícolas, por meio da sua introdução na adubação das plantas ou por melhoramento genético, visando atender à necessidade humana ou animal (Graham et al., 2007; Cakmak,
2008). Nesse sentido, diversos autores verificaram que o teor de Se em culturas agrícolas aumentou com a introdução desse elemento na adubação, demonstrando ser uma técnica útil para elevar a ingestão de Se pela população (Chen et al., 2002; Ríos et al., 2008; Broadley et al., 2010). No Brasil, há poucas informações a respeito da aplicação de Se em plantas, todavia são grandes as evidências de deficiência desse elemento em algumas regiões do País (Maihara et al., 2004; Moraes et al., 2009). Além disso, Ferreira et al. (2002) relataram que a concentração de Se nos alimentos de origem vegetal consumidos no Brasil é considerada baixa, em relação aos padrões internacionais.

Nesse contexto, devido à importância de se estudar a biofortificação de culturas agrícolas com Se, associada à escassez de informações a esse respeito no Brasil, o objetivo deste trabalho foi avaliar o efeito da aplicação de selenato e selenito na biofortificação com Se, bem como verificar a influência dessas formas de Se nos teores de macro e micronutrientes, em cinco cultivares de alface.

\section{MATERIAL E MÉTODOS}

O experimento foi conduzido em casa de vegetação no Departamento de Ciência do Solo da Universidade Federal de Lavras. Utilizou-se o delineamento experimental inteiramente casualizado, em esquema fatorial $5 \times 3 \times 2$, sendo cinco cultivares de alface (Maravilha de Verão, Rafaela, Great Lakes, Veneranda e Vera), três concentrações de Se na solução nutritiva $\left(0,10\right.$ e $\left.20 \mu \mathrm{mol} \mathrm{L}^{-1}\right)$ e duas formas de Se (selenato de sódio $-\mathrm{Na}_{2} \mathrm{SeO}_{4}$, e selenito de sódio $-\mathrm{Na}_{2} \mathrm{SeO}_{3} .5 \mathrm{H}_{2} \mathrm{O}$, 
ambos reagentes Sigma-Aldrich), com quatro repetições, perfazendo um total de 120 parcelas. Cada unidade experimental foi constituída por uma planta de cada cultivar por vaso.

As sementes de alface foram semeadas em bandejas de poliestireno expandido contendo 128 células, preenchidas com vermiculita e irrigadas com água destilada nos primeiros cinco dias. A partir do sexto dia, as plântulas foram irrigadas com solução nutritiva de Hoagland (Hoagland \& Arnon, 1950) com $5 \%$ da força iônica. Posteriormente, as mudas foram selecionadas quanto ao vigor e uniformidade, tendo suas raízes lavadas com água destilada, e transplantadas para vasos de $3 \mathrm{~L}$ contendo solução nutritiva de Hoagland com 40 \% da força iônica, quando então foram aplicados os respectivos tratamentos de Se.

Durante todo o período experimental, a solução de cultivo foi submetida à aeração constante e o pH monitorado diariamente, mantendo-se em 5,8-6,0 pela correção com $\mathrm{NaOH}$ ou $\mathrm{HCl} 0,1 \mathrm{~mol} \mathrm{~L}^{-1}$. A troca da solução nutritiva foi realizada quando o valor da condutividade elétrica inicial foi reduzido em $30 \%$. Após 25 dias de exposição às formas de Se, procedeuse à colheita das plantas, separando-as em parte aérea e raiz. Posteriormente, cada parte foi lavada em água corrente, seguida por água bidestilada, quatro vezes e conduzida para estufa de circulação forçada de $\operatorname{ar}\left(50^{\circ} \mathrm{C}\right)$, até atingir peso constante. Em seguida, essas partes foram trituradas e submetidas à análise química para determinação dos teores de Se, de acordo com Ramos et al. (2010). Para certificação dos teores de Se, foi utilizado material certificado (Tomato Leaf, NIST 1573a), o qual foi incluído em cada bateria de digestão. Determinaram-se, também, os teores de macro e micronutrientes na parte aérea, conforme Malavolta et al. (1997).

Relacionando os teores de Se com a massa seca produzida, foi determinado o acúmulo de Se na parte aérea e em toda a planta (parte aérea + raiz). Uma vez obtidos esses dados, calculou-se o transporte do Se para a parte aérea, utilizando a seguinte expressão: [(mg de Se acumulado na parte aérea) / (mg de Se acumulado na planta) x 100].

Os dados foram submetidos à análise de variância e testes de média, com o auxílio do programa estatístico Sisvar (Ferreira, 1999). As médias dos tratamentos componentes do fatorial foram comparadas entre si pelo teste de Scott-Knott ( $p \leq 0,05)$.

\section{RESULTADOS E DISCUSSÃO}

A produção de massa seca da parte aérea e raiz, o transporte e o teor de Se na parte aérea foram influenciados significativamente pelas cultivares de alface, pelas concentrações de Se e pelas formas de Se na solução nutritiva, bem como pela interação entre esses fatores (Figura 1). Com exceção do tratamento controle ( $0 \mu \mathrm{mol} \mathrm{L}{ }^{-1}$ de Se), os valores da massa seca da parte aérea, independentemente da cultivar, foram superiores quando se utilizou o Se na forma de selenato (Figura 1a,b). Para a massa seca da raiz, apenas a cultivar Great Lakes nas concentracões de $10 \mathrm{e}$ $20 \mu \mathrm{mol} \mathrm{L}{ }^{-1}$ apresentou diferença significativa entre as formas de Se utilizadas, sendo o selenato superior ao selenito (Figura 1c,d). Para todas as cultivares, o aumento das concentrações de Se de 10 para $20 \mu \mathrm{mol} \mathrm{L}{ }^{-1}$, em ambas as formas de Se aplicadas, diminuiu a produção de massa seca da parte aérea e raiz, à exceção da cultivar Veneranda, para parte aérea e raiz (Figura 1a, c), e Vera e Rafaela, para a raiz (Figura 1c), quando cultivadas na presença do selenato. Essa redução na produção da parte aérea e raiz pode estar relacionada à substituição do enxofre (S) na cisteína e metionina, formando selenocisteína e selenometionina. Segundo Zhu et al. (2009), essa substituição ocorre devido à semelhança estrutural entre o Se e o S e altera a síntese e função de aminoácidos e proteínas nas plantas. Além disso, Pazurkiewicz-Kocot et al. (2008) relataram que o Se no interior da planta atua como análogo do $\mathrm{S}$, interferindo nas reações bioquímicas celulares.

Os resultados encontrados para a parte aérea mostram, de maneira geral, que as cultivares de alface apresentaram maior tolerância para o Se na forma de selenato, sendo o selenito mais tóxico. O efeito diverso do selenato e selenito no crescimento das cultivares de alface pode ser causa dos distintos mecanismos de metabolismo das diferentes formas de Se (Sors et al., 2005). Nesse sentido, Hopper \& Parker (1999) relataram que o selenito é rapidamente convertido em formas orgânicas de Se, as quais são incorporadas em proteínas em substituição ao S, causando toxidez mais rapidamente quando comparado ao selenato. Os resultados encontrados no presente estudo são semelhantes ao observado por Ríos et al. (2008), ao avaliarem a biofortificação com Se e a indução da capacidade antioxidante em plantas de alface utilizando o selenato e selenito, e ao verificado por Ramos et al. (2011), ao estudarem a variação genotípica em diversos germoplasmas de alface.

Alguns estudos mencionam que o selenato e o selenito proporcionam distintas respostas na translocação do Se das raízes para a parte aérea (Zhang et al., 2003). Dessa forma, verifica-se que a translocação do Se foi sempre maior quando fornecido na forma de selenato (Figura 1e,f). Nos tratamentos que receberam as concentrações de 10 e $20 \mu \mathrm{mol} \mathrm{L}^{-1}$ de Se, em média, cerca de 80 e $50 \%$ do elemento aplicado foram translocados para a parte aérea, para o selenato e selenito, respectivamente. Esses resultados são semelhantes aos obtidos em outros trabalhos, os quais relatam superioridade do selenato sobre o selenito no transporte de Se pelas plantas (Chen et al., 2002; Zhang et al., 2003; Cartes et al., 2005). Li et al. (2008) relataram que o Se do selenito, quando absorvido pelas plantas, é rapidamente convertido em 

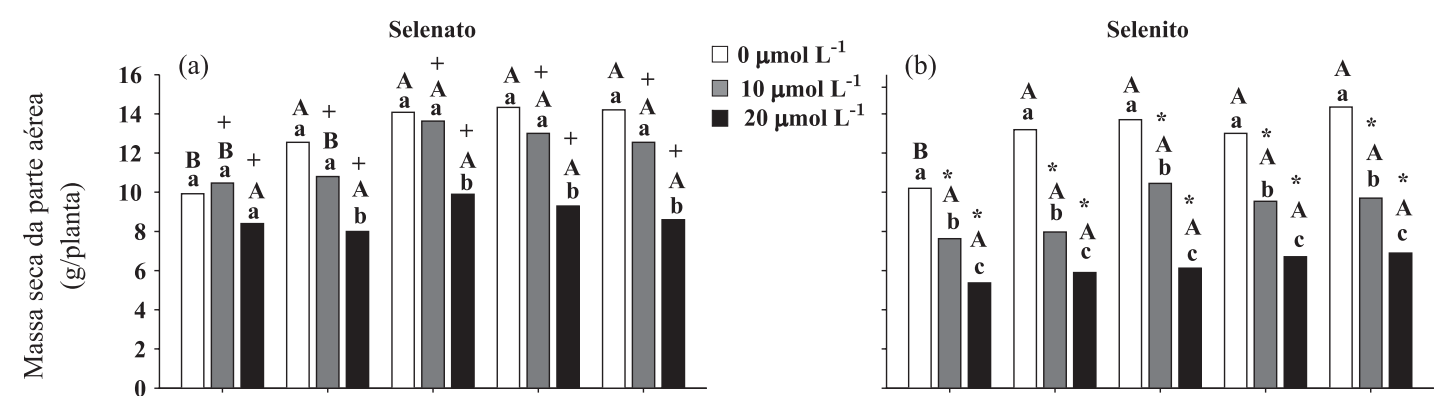

(c)

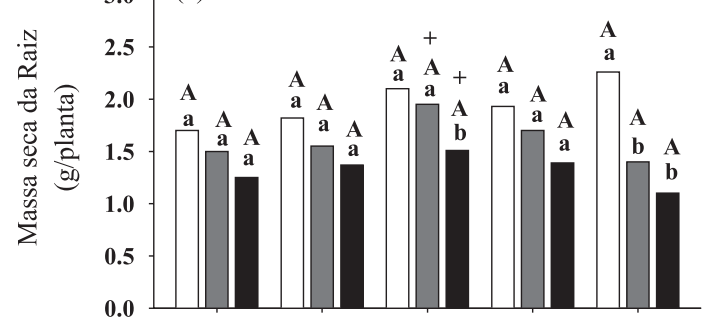

(d)
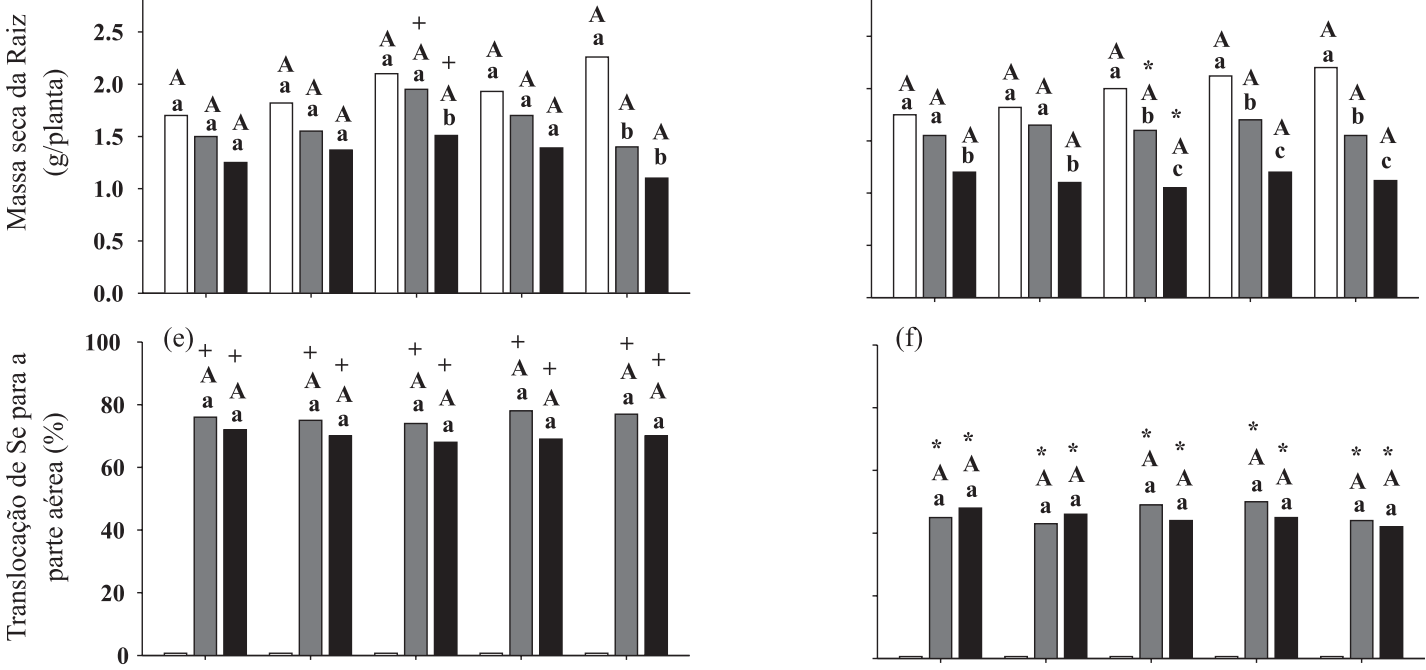

(f)
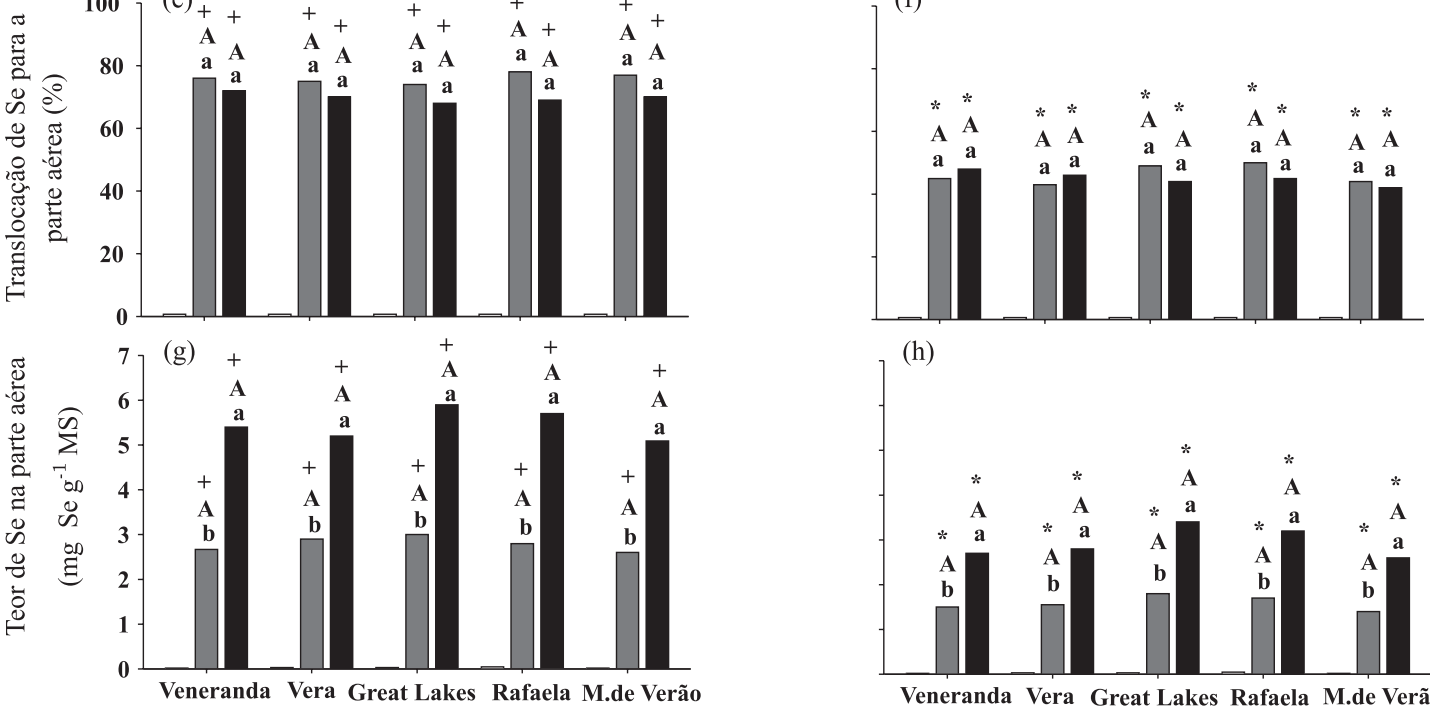

(h)

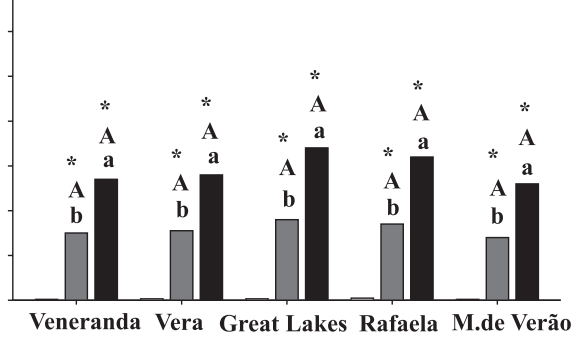

Figura 1. Massa seca da parte aérea $(a, b)$, da raiz $(c, d)$, translocação de Se para a parte aérea (e,f) e teor de Se na parte aérea $(g, h)$ de cultivares de alface em função da aplicação de diferentes concentrações e formas de Se. Médias seguidas de mesma letra minúscula, comparando concentrações de Se dentro de cada cultivar, e maiúscula, comparando as cultivares em cada concentração e forma de Se, não diferem entre si (Scott-Knott, $p \leq 0,05)$. Médias seguidas por símbolos diferentes $(+\mathrm{e} *$ ), comparando as formas de Se (selenato e selenito) dentro de cada concentração de Se e cultivar, diferem entre si (Scott-Knott,

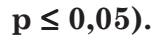

formas orgânicas, as quais apresentam baixa mobilidade no xilema; em contrapartida, o selenato nas raízes não é facilmente convertido em formas orgânicas, sendo altamente móvel no xilema. Ríos et al. (2008) e Ramos et al. (2010) também verificaram que, para uma mesma concentração de Se aplicada, o fornecimento de selenato proporcionou maior translocação e elevou acúmulo de Se na parte aérea em plantas de alface.

Segundo Terry et al. (2000), as formas de Se proporcionam diferentes teores de Se nas plantas. Nesse sentido, verificou-se que o teor foliar de Se aumentou com o aumento da sua concentração em solução, para todas as cultivares e formas de Se (Figura 1g,h). Independentemente da concentração aplicada, os teores foliares, em todas as cultivares, foram superiores quando se utilizou o Se na forma de selenato (Figura 1g,h). Isso se deve à maior mobilidade do Se nessa forma, como comentado anteriormente e mostrado na figura 1e,f, cujos resultados concordam com os observados por Chen et al. (2002), Cartes et al. (2005), Ríos et al. (2008) e Ramos et al. (2010).

O que se busca em programas de biofortificação com Se é elevar o seu teor, sem comprometer a 
produção agrícola. Nesse sentido, observou-se que a biofortificação com Se nas cultivares de alface dependeu da concentração e da forma de Se aplicada. $\mathrm{O}$ selenato na concentração de $10 \mu \mathrm{mol} \mathrm{L}^{-1}$ não comprometeu a produção da parte aérea (Figura 1a) e, ainda, promoveu aumento no teor de Se, em todas as cultivares (Figura 1g). Por sua vez, o selenito, independentemente da concentração e da cultivar, comprometeu a produção de massa seca da parte aérea e foi sempre inferior na biofortificação com Se (Figura 1g,h). Por esse resultado, infere-se que o selenato se apresentou mais eficiente na biofortificação das cultivares de alface avaliadas, podendo ser utilizado de forma mais efetiva para aumentar a ingestão desse elemento em humanos.

Levando-se em conta que no Brasil o consumo per capta de folhas frescas de alface é de $33,3 \mathrm{~g} \mathrm{dia}^{-1}$ (Yuri et al., 2005), que a alface apresenta $4 \%$ de matéria seca (Faquin \& Andrade, 2004) e que a recomendação de ingestão de Se em adultos é de 50-70 $\mu \mathrm{g}$ dia $^{-1}$ (Ríos et al., 2008), verificou-se que o teor encontrado de Se na parte aérea, quando se aplicou o selenato na concentração de $10 \mu \mathrm{mol} \mathrm{L}{ }^{-1}$, a qual não comprometeu a produção, atendeu em aproximadamente $5 \%$ à recomendação de ingestão de Se em humanos, independentemente da cultivar de alface. Esses resultados são inferiores aos encontrados por Ríos et al. (2008). Dessa forma, sugere-se, a partir deste estudo, incrementar as pesquisas sobre como biofortificar diferentes culturas com Se, a fim de aumentar a ingestão de Se na população brasileira, a qual apresenta indicativo de baixo consumo desse elemento (Ferreira et al., 2002; Maihara et al., 2004; Moraes et al., 2009). A isso soma-se o fato de que melhorar a concentração de micronutrientes tornouse uma tarefa urgente, pois cerca de metade da população mundial sofre com a desnutrição de $\mathrm{Fe}, \mathrm{Zn}$ e Se (Zhao et al., 2009).

Estudos relatam que o selenato utiliza os mesmos transportadores de membrana do sulfato (Terry et al., 2000; Sors et al., 2005), e o Se utiliza a mesma rota de assimilação do S (Pilon-Smits \& Quinn, 2010). Assim, alterações na absorção e no teor de $\mathrm{S}$ promovidas pelo Se são esperadas, tal como observado por Smith \& Watkinson (1984), Banuelos et al. (1990), Mikkelsen \& Wan (1990) e Barack \& Goldman (1997). No presente estudo, o aumento nas concentrações de selenato aumentou o teor de $\mathrm{S}$ em todas as cultivares de alface, tendo a maior concentração proporcionado os maiores teores de S (Figura 2a). Por outro lado, não se observou diferença entre as concentrações e cultivares quando se utilizou o Se na forma de selenito (Figura 2b). Mikkelsen \& Wan (1990), ao aumentarem a concentração de selenato na solução de cultivo, também verificaram aumento significativo no teor de S na parte aérea de cevada e arroz. Esses autores relatam que a interação sinérgica entre o Se e o S pode ocorrer em muitas espécies vegetais. Kopsell et al. (2000), em Brassica oleraceae, White et al.
(2004), em Arabidopsis thaliana, e Ramos et al. (2011), em germoplasmas de alface, também observaram resultados semelhantes aos do presente trabalho.

Entretanto, vale ressaltar que as primeiras pesquisas visando estudar o efeito do Se na nutrição mineral de plantas relataram que o selenato inibia a absorção de sulfato, o que promovia diminuição do teor de S no tecido vegetal (Ferrari \& Renosto, 1972). No entanto, nesse trabalho foram utilizadas proporções equivalentes de selenato e sulfato na solução de cultivo. Essa inibição também foi observada por Barack \& Goldman (1997), onde a alta disponibilidade de selenato diminuiu o teor de S na cultura da cebola, o que pode ser atribuído, segundo esses autores, à inibição competitiva com o sulfato, promovida pelo excesso de selenato em solução de cultivo. Assim, o presente estudo mostra que o selenato em menores concentrações que o sulfato favoreceu o aumento dos teores de $\mathrm{S}$ nas cultivares de alface avaliadas.

Os teores de P na parte aérea das cultivares de alface foram reduzidos pela aplicação de selenito na solução, mesmo na concentração de $10 \mu \mathrm{mol} \mathrm{L}^{-1}$; esse fato não foi observado quando se utilizou o selenato (Figura 2c,d), mostrando uma possível inibição competitiva do selenito na absorção de $\mathrm{P}$, resultando em menor teor desse elemento nas plantas. Hopper \& Parker (1999) observaram inibição competitiva entre o selenito e o P. Segundo esses autores, o aumento nas concentrações de selenito ou de fosfato na solução de cultivo pode promover alterações nos teores desses elementos em diversas plantas. Esse fato foi observado por Liu et al. (2004), em cujo estudo o suprimento de $\mathrm{P}$ em condições normais para o crescimento do arroz não alterou a absorção e o teor de Se nessa cultura. Entretanto, com o aumento da disponibilidade de P na solução de cultivo, esses autores verificaram redução no teor de Se, em todas as concentrações de selenito utilizadas.

$\mathrm{O}$ aumento nas concentrações de ambas as formas de Se não alterou os teores de $\mathrm{Mg}$ (Figura 2e,f) e de Ca (Figura 2g,h), à exceção da Veneranda para o Ca em ambas as formas de Se, em que o aumento da concentração deste na solução reduziu o teor daquele na cultivar. Em milho, Pazurkiewicz-Kocot et al. (2008), com selenito, e Hawrylak-Nowak (2008), com selenato, também não observaram efeito das formas de Se nos teores de Mg na cultura. Kopsell et al. (2000) também não observaram efeitos do selenato na solução de cultivo sobre os teores de Ca em Brassica oleracea

De maneira geral, verificou-se que o aumento das concentrações de ambas as formas de Se diminuiu os teores de micronutrientes em todas as cultivares de alface (Figura 3). Comparando-se as formas de Se, apenas para o Mn na cultivar Vera (Figura 3c,d) e para o Fe na cultivar M. de Verão (Figura 3e,f), o selenato proporcionou teores mais elevados que os de selenito. Feng et al. (2009), ao aplicarem selenito em 

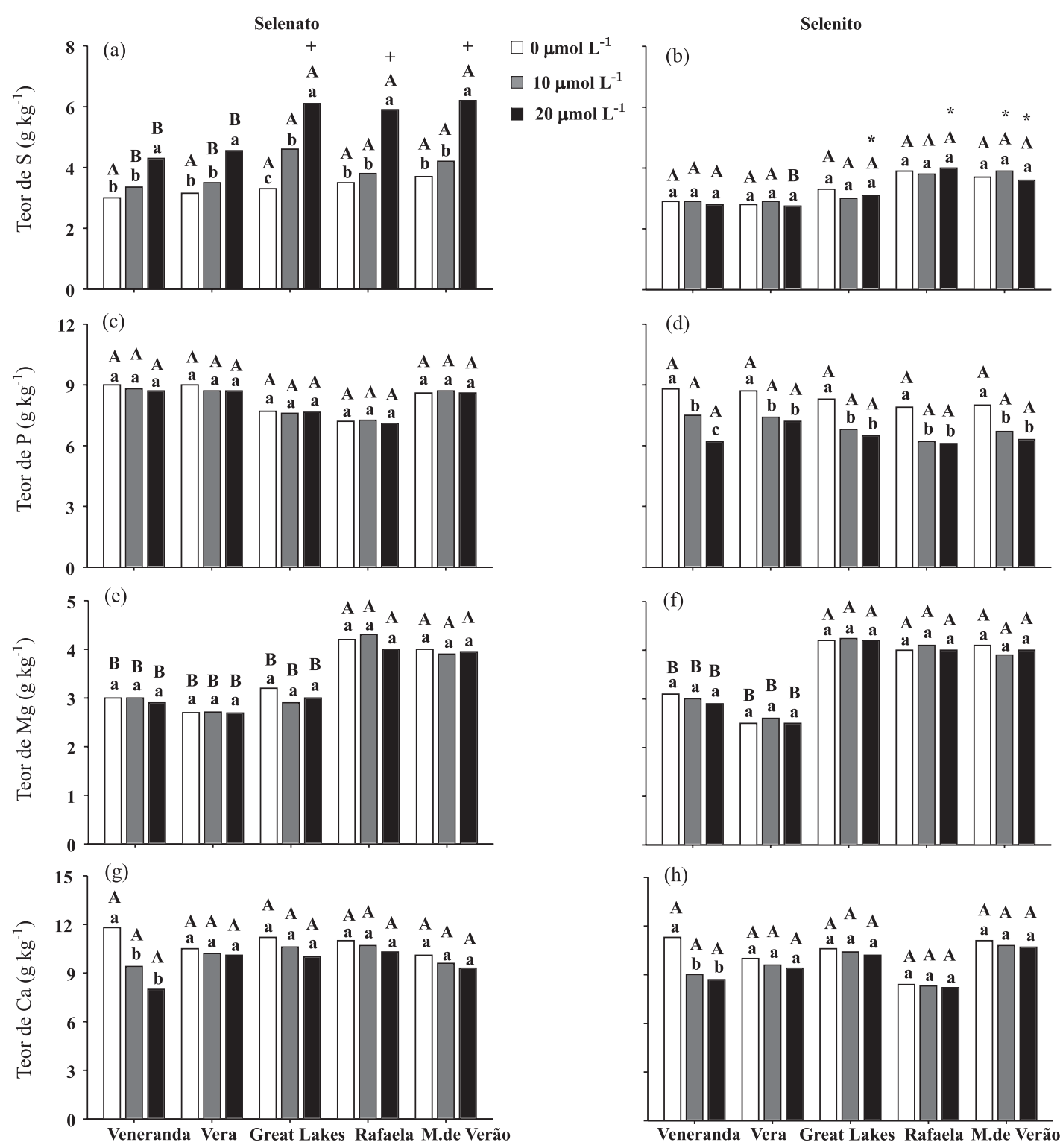

Figura 2. Teores de S (a, b), P (c, d), Mg (e,f) e Ca $(g, h)$ na parte aérea de cultivares de alface, em função da aplicação de diferentes concentrações e formas de Se. Médias seguidas de mesma letra minúscula, comparando concentrações de Se dentro de cada cultivar, e maiúscula, comparando as cultivares em cada concentração e forma de Se, não diferem entre si (Scott-Knott, $p \leq 0,05)$. Médias seguidas por símbolos diferentes $\left(+e^{*}\right)$, comparando as formas de Se (selenato e selenito) dentro de cada concentração de Se e cultivar, diferem entre si (Scott-Knott, $p \leq 0,05)$.

Pteris vittata cultivada em solução nutritiva e em solo, também observaram que menores doses de Se diminuíram os teores de $\mathrm{Cu}, \mathrm{Mn}, \mathrm{Fe}$ e Zn, em ambos os sistemas de cultivo. A notável diminuição nos teores desses micronutrientes deve-se, segundo esses autores, ao efeito antagônico do Se para esses elementos. Da mesma forma, Pazurkiewicz-Kocot et al. (2008) verificaram diminuição nos teores de $\mathrm{Cu}$ e $\mathrm{Mn}$, em folhas de milho, quando adicionaram selenito na adubação e relataram que a presença do Se no interior vegetal pode alterar o coeficiente de permeabilidade de íons na membrana plasmática e, consequentemente, o transporte e o acúmulo de micronutrientes nas células vegetais. De acordo com esses autores, a mudança de capacidade de transporte de íons e seus efeitos no acúmulo de nutrientes são, provavelmente, um dos primeiros sintomas observados dos efeitos de Se nas plantas.

Dessa maneira, este estudo mostrou a importância de aumentar o teor de Se em culturas agrícolas, a fim de proporcionar o aumento na ingestão desse elemento em humanos. Entretanto, essa estratégia pode influenciar os teores de macro e micronutrientes nas plantas e alterar o equilíbrio nutricional delas. 

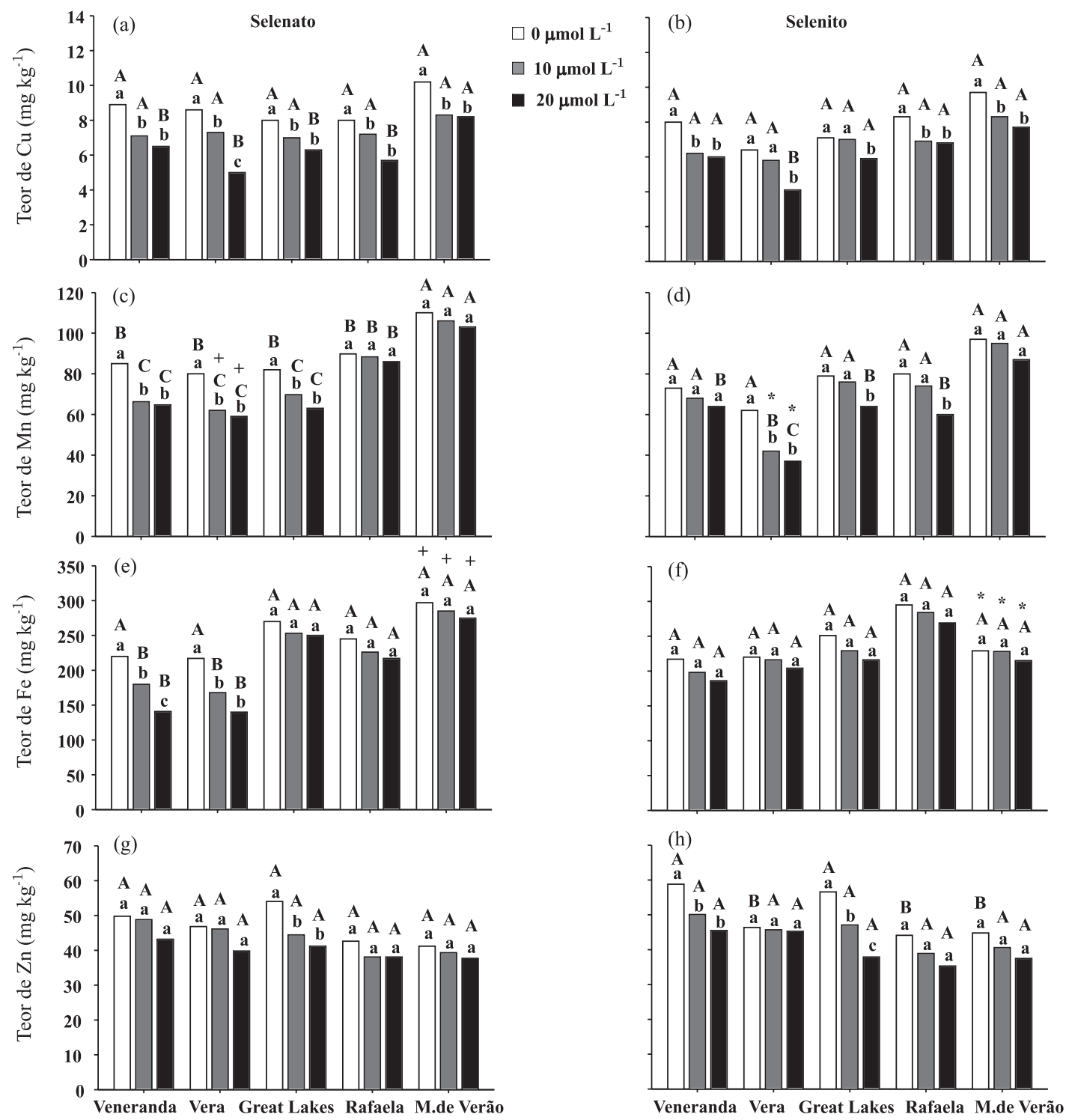

Figura 3. Teores de Cu (a e b), Mn (c e d), Fe (e e f) e Zn (g e h) na parte aérea de cultivares de alface, em função da aplicação de diferentes concentrações e formas de Se. Médias seguidas de mesma letra minúscula, comparando concentrações de Se dentro de cada cultivar, e maiúscula, comparando as cultivares em cada concentração e forma de Se, não diferem entre si (Scott-Knott, p $\leq 0,05)$. Médias seguidas por símbolos diferentes $\left(+e^{*}\right)$, comparando as formas de Se (selenato e selenito) dentro de cada concentração de Se e cultivar, diferem entre si (Scott-Knott, p $\leq$ 0,05).

\section{CONCLUSÕES}

1. A aplicação do Se na forma de selenato mostrou ser mais indicada para a biofortificação em cultivares de alface, enquanto o selenito mostrou ser mais tóxico.

2. A aplicação do selenato resultou em aumento no teor de S na parte aérea, enquanto o selenito reduziu o teor de $\mathrm{P}$, e ambas as formas de Se diminuíram os teores de micronutrientes.

3. Entre as cultivares de alface utilizadas, não se observou variação genotípica para o teor de Se, e pequena variação foi verificada para a produção de matéria seca da parte aérea e teores de S, Mg, Mn e Fe.

\section{AGRADECIMENTOS}

Ao CNPq e FAPEMIG pela concessão de bolsa de produtividade, mestrado, doutorado e pós-doutorado.

\section{LITERATURA CITADA}

BANUELOS, G.S.; MEEK, D.W. \& HOFFMAN, G.J. The influence of selenium, salinity, and boron on selenium uptake in wild mustard. Plant Soil, 127:201-206, 1990. 
BARACK, P. \& GOLDMAN, I.L. Antagonistic relationship between selenate and sulfate uptake in onion (Allium cepa): implications for the production of organosulfur and organoselenium compounds in plants. J. Agric. Food. Chem., 45:1290-1294, 1997.

BROADLEY, M.R.; ALCOCK, J.; ALFORD, J.; CARTWRIGHT, P.; FOOT, I.; FAIRWEATHER-TAIT, S.J.; HART, D.J.; HURST, R.; KNOTT, P.; MCGRATH, S.P.; MEACHAM, M.C.; NORMAN, K.; MOWAT, H.; SCOTT, P.; STROUD, J.L.; TOVEY, M.; TUCKER, M.; WHITE, P.J.; YOUNG, S.D. \& ZHAO, F.J. Selenium biofortification of highyielding winter wheat (Triticum aestivum L.) by liquid or granular Se fertilisation. Plant Soil, 332:5-18, 2010.

CAKMAK, I. Enrichment of cereal grains with zinc: Agronomic or genetic biofortification? Plant Soil, 302:1-17, 2008.

CARTES, P.; GIANFREDA, L. \& MORA, M.L. Uptake of selenium and its antioxidant activity in ryegrass when applied as selenate and selenite forms. Plant Soil, 276:359$367,2005$.

CHEN, L.; YANG, F.; XU, J.; YUN, H.; HU, Q.; ZHANG, Y. \& PAN, G. Determination of selenium concentration of rice in China and effect of fertilization of selenite and selenate on Se content of rice. J. Agric. Food. Chem., 50:5128-5130, 2002.

FAIRWEATHER-TAIT, S.; BAO, Y.; BROADLEY, M.; COLLINGS, R.; FORD, D.; HESKETH, J. \& HURST, R. Selenium in human health and disease. Antioxid. Redox Signaling, 14:1337-1383, 2011.

FAQUIN, V. \& ANDRADE, A.T. Nutrição mineral e diagnose do estado nutricional de hortaliças. Lavras, UFLA/FAEPE, 2004. 88p.

FENG, R.; WEI, C.; TU, S. \& WU, F. Effects of Se on the uptake of essential elements in Pteris vittata L. Plant Soil, 325:123-132, 2009.

FERRARI, G. \& RENOSTO, F. Regulation of sulfate uptake by excised barley roots in the presence of $\mathrm{SeO}_{4}$. Plant Physiol., 49:114-116, 1972.

FERREIRA, D.F. Sistema para análise de variância para dados balanceados (SISVAR). Lavras, Universidade Federal de Lavras, 1999. 92p.

FERREIRA, K.S.; GOMES, J.C.; BELLATO, C.R. \& JORDÃO, C.P. Concentrações de selênio em alimentos consumidos no Brasil. R. Panam. Salud Publica, 11:172-177, 2002.

GRAHAM, R.D.; WELCH, R.M.; SAUNDERS, D.A.; ORTIZMONASTERIO, I.; BOUIS, H.E.; BONIERBALE, M.; DE HAAN, S.; BURGOS, G.; THIELE, G.; LIRIA, R.; MEISNER, C.A.; BEEBE, S.E.; POTTS, M.J.; KADIAN, M.; HOBBS, P.R.; GUPTA, R.K. \& TWOMLOW, S. Nutritious subsistence food systems. Adv. Agron., 92:1$74,2007$.

HARTIKAINEN, H.; XUE, T.L. \& PIIRONEN, V. Selenium as an antioxidant and pro-oxidant in ryegrass. Plant Soil, 225:193-200, 2000.

HAWRYLAK-NOWAK, B. Effect of selenium on selected macronutrients in maize plants. J. Element., 13:513-519, 2008.
HOAGLAND, D.R. \& ARNON, D. The water culture method for growing plants without soil. California Agric. Exper. Sta. Circ., 374:1-32, 1950.

HOPPER, J. \& PARKER, D. Plant availability of selenite and selenate as influenced by the competing ions phosphate and sulfate. Plant Soil, 210:199-207, 1999.

KOPSELL, D.A.; RANDLE, W.M. \& MILLS, H.A. Nutrient accumulation in leaf tissue of rapid-cycling Brassica oleracea responds to increasing sodium selenate concentrations. J. Plant Nutr., 23:927-935, 2000.

LI, H.; MCGRATH, S. \& ZHAO, F. Selenium uptake, translocation and speciation in wheat supplied with selenate or selenite. New Phytol., 178:92-102, 2008.

LIU, Q.; WANG, D.J.; JIANG, X.J. \& CAO, Z.H. Effects of the interactions between selenium and phosphorus on the growth and selenium accumulation in rice (Oryza sativa). Environ. Geochem. Health., 26:325-330, 2004.

LYONS, G.H.; GENC, Y.; SOOLE, K.; STANGOULIS, J.C.R.; LIU, F. \& GRAHAM, R.D. Selenium increases seed production in Brassica. Plant Soil, 318:73-80, 2009.

MAIHARA, V.A.; GONZAGA, I.B.; SILVA, V.L.; FÁVARO, D.I.T.; VASCONCELLOS, M.B.A. \& COZZOLINO, S.M.F. Daily dietary selenium intake of selected Brazilian population groups. J. Radio. Nucl. Chem., 259:465-468, 2004.

MALAVOLTA, E.; VITTI, G.C. \& OLIVEIRA, S.A. Avaliação do estado nutricional das plantas: Princípios e aplicações. 2.ed. Piracicaba, Potafos, 1997. 319p.

MIKKELSEN, R.L. \& WAN, H.F. The effect of selenium on sulfur uptake by barley and rice. Plant Soil, 121:151-153, 1990.

MORAES, M.F.; WELCH, R.M.; NUTTI, M.R.; CARVALHO, J.L.V. \& WATANABE, E. Evidences of selenium deficiency in Brazil: From soil to human nutrition. In: INTERNATIONAL CONFERENCE ON SELENIUM IN THE ENVIRONMENT AND HUMAN HEALTH, 1., Suzhou, 2009. Proceedings... Suzhou, 2009. p.73-74.

PAZURKIEWICZ-KOCOT, K.; KITA, A. \& ANDPIETRUSZKA, M. Effect of selenium on magnesium, iron, manganese, copper, and zinc accumulation in corn treated by indole-3-acetic acid. Comm. Soil Sci. Plant Anal., 39:2303-2318, 2008.

PILON-SMITS, E. \& QUINN, C. Selenium metabolism in plants. In: HELL, R. \& MENDEL, R.R., eds. Cell biology of metal and nutrients. Berlin, Springer, 2010. p.225-241.

RAMOS, S.J.; RUTZKE, M.A.; HAYNES, R.J.; FAQUIN, V.; GUILHERME, L.R.G. \& LI, L. Selenium accumulation in lettuce germplasm. Planta, 233:649-660, 2011.

RAMOS, S.J.; FAQUIN, V.; GUILHERME, L.R.G.; CASTRO, E.M.; AVILA, F.W.; CARVALHO, G.S.; BASTOS, C.E.A. \& OLIVEIRA, C. Selenium biofortification and antioxidant activity in lettuce plants fed with selenate and selenite. Plant Soil Environ., 12:583-587, 2010.

RAYMAN, M.P. The argument for increasing selenium intake. Proc. Nutr. Soc., 61:203-215, 2002 
RÍOS, J.J.; ROSALES, M.A.; BLASCO, B.; CERVILHA, L.; ROMERO, L. \& RUIZ, J.M. Biofortification of Se and induction of the antioxidant capacity in lettuce plants. Sci. Hortic., 116:248-255, 2008.

SMITH, G.S. \& WATKINSON, J.H. Selenium toxicity in perennial ryegrass and white clover. New Phytol., 97:557564,1984

SORS, T.G.; ELLIS, D.R. \& SALT, D.E. Selenium uptake, translocation, assimilation and metabolic fate in plants. Photosynth. Res., 86:373-389, 2005.

STRATTON, M.S.; REID, M.E.; SCHWARTZBERG, G.; MINTER, F.E.; MONROE, B.K.; ALBERTS, D.S.; MARSHALL, J.R. \& AHMANN, F.R. Selenium and prevention of prostate cancer in high-risk men: The negative biopsy study. Anticancer. Drugs., 14:589-594, 2003.

TERRY, N.; ZAYED, A.M.; SOUZA, M.P. \& TARUN, A.S Selenium in higher plants. Ann. Rev. Plant Physiol. Plant Molec. Biol., 51:401-432, 2000.
WHITE, P.J.; BOWEN, H.C.; PARMAGURU, P.; FRITZ, M.; SPRACKLEN, W.P.; SPIBY, R.E.; MEACHAM, M.C.; MEAD, A.; HARRIMAN, M.; TRUEMAN, L.J.; SMITH, B.M.; THOMAS, B. \& BROADLEY, M.R. Interactions between selenium and sulphur nutrition in Arabidopsis thaliana. J. Exper. Bot., 55:1927-1937, 2004.

YURI, J.E.; SOUZA, R.J.; RESENDE, G.M. \& MOTA, J.H. Comportamento de cultivares de alface americana em Santo Antônio do Amparo. Hortic. Bras., 23:870-874, 2005.

ZHANG, Y.; PAN, G.; CHEN, J. \& HU, Q. Uptake and transport of selenite and selenate by soybean seedlings of two genotypes. Plant Soil, 253:437-443, 2003.

ZHAO, F.J.; SU, Y.H.; DUNHAM, S.J.; RAKSZEGI, M.; BEDO, Z.; McGRATH, S.P. \& SHEWRY, P.R. Variation in mineral micronutrient concentrations in grain of wheat lines of diverse origin. J. Cereal Sci., 49:290-295, 2009.

ZHU, Y.G.; PILON-SMITS, E.A.H.; ZHAO, F.J.; WILLIAMS, P.N. \& MEHARG, A.A. Selenium in higher plants: Understanding mechanisms for biofortification and phytoremediaton. Trends Plant Sci., 14:436-442, 2009. 
